\title{
PINK DISEASE : ITS MORBID ANATOMY, WITH A NOTE ON TREATMENT
}

BY

W. G. WYLLIE, M.D., M.R.C.P., and R. O. STERN, M.B., B.S.

(From the Hospital for Sick Children, Great Ormond Street, and the Laboratory of the National Hospital, Queen Square, London.)

In its clinical aspect, pink disease has received a great amount of attention, as can be seen from the abundant case reports in the literature. Its pathological investigation, however, has been served less well, for, although most of the symptoms point to a nervous disorder, very few autopsies have included a microscopic examination of the nervous system. The main object, therefore, of this paper is to record the results of the histological examination of the nervous system in seven cases of pink disease.

The pathogenesis of the disease remains unrecognized, but various views as to its causation will be touched upon. Lastly, we offer a few notes on the results of a certain form of treatment which appears to be of definite value.

Historical.-It is difficult to say if we are dealing with a new disease. Certainly in the literature no mention of the condition can be found earlier than 1903 , but various authorities have remarked that they were aware of its clinical features prior to that date.

The first mention of the disease appears to have been made in 1903 by Selter ${ }^{1}$, who described eight cases at a medical society meeting for children's diseases at Cassel (Germany). Since then one or two of his cases have been regarded as doubtful. The title employed by Selter for the condition was 'trophodermatoneurose' or 'vegetative neurosis.' 'This early communication, however, escaped attention, and a wider interest was not aroused until several years later when many cases were described in Australia.

In Australia, Swift ${ }^{2}$ of Adelaide in 1914 reported 14 cases under the title of 'erythrodema.' Between 1917 and 1920, Wood and Cole ${ }^{3}$ of Melbourne collected 91 cases, and Wood remarked that he had 'frequently seen . . . cases in Melbourne for the past thirty years.' In Sydney, Clubbe, Littlejohn ${ }^{4}$ (1923), and others had for many years been familiar with the condition, and were in the habit of referring to it as 'the pink disease.'

In America, Byfield ${ }^{5}$ (1917 and 1920), and Bilderback ${ }^{6}$ (1919), recorded numerous cases, which were soon followed by the reports of many other observers. The earliest examples to be described in Great Britain were by Parkes Weber ${ }^{7}$ in 1921, and Thursfield and Paterson ${ }^{8}$ in 1922 (dermato-polyneuritis). In Germany for the second time the syndrome was described, on this occasion by Feer' in 1923. In France, Haushalter ${ }^{10}$ (1925), wrote on a 'neuro-vegetative syndrome' in infants, and mentioned that he had been aware of the condition since 1911. The disease has also been recorded in several other European countries.

For the sake of simplicity the commonplace name of 'pink disease' will be employed in this paper, though the terms 'trophodermatoneurose' of Selter, and 'neurosis of the vegetative nervous system' of Feer, are of value 
in indicating that the symptoms are mainly related to the vegetative nervous system. Erythrœdema is an unfortunate title, as there is no true œdema of the parts affected.

Symptomatology.-The syndrome known as pink disease wears such a distinctive stamp that the diagnosis is usually apparent at a glance. Con. spicuous symptoms are irritability, photophobia, bluish-red discolouration with slight swelling of the hands and feet-' raw-beef hands and feet'-excessive sweating, muscular hypotonicity, and in some chronic cases sensory impairment and loss of the tendon jerks.

The disease is one of infancy and early childhood, affecting both sexes equally, most cases occurring between the ninth and eighteenth months. Infants as young as four months are not infrequently affected, and more rarely, children up to the seventh year. The youngest case we have personally observed was a breast-fed infant of ten weeks, in whom the symptoms com. menced in the third week after birth. It occurs in the breast-fed as well as in the artificially-fed infant, and both in rural and urban areas. The time of year in which the greater number of cases are met with is between autumn and early spring. Most cases are sporadic, and there is no evidence that the condition is infectious. Nevertheless, a tendency to grouping of cases by locality suggestive of small epidemics has been noted by several observers (foyers epidemiques, inseln).

The sequence of events in a typical case is as follows:- Soon after some respiratory or intestinal catarrh, or without prodromal symptoms of infection, the child becomes acutely miserable and irritable, sleep is disturbed, appetite reduced, photophobia appears, and the hands and feet become cyanosed, bluish-red, slightly swollen, glazed, and very cold. A pink erythematous patch is usually present on the point of the nose and both cheeks. Rashes of a papulo-macular type occur on the body, but most often on the extremities only, reaching as far up the limbs as the wrists and ankles. Sweating is a prominent symptom, and may be extreme, and is frequently the cause of a miliary sudaminal rash covering the body. The pulse rate is accelerated, commonly to between 120 and 160 beats per minute, and in many cases the blood pressure is raised (110 to $130 \mathrm{~mm}$.). Despite the sweating and quickened pulse the temperature is always normal in an uncomplicated case.

A characteristic position in bed is often assumed, the knee-elbow position, in which the child rubs and burrows its face in the pillow, at the same time keeping the light from its eyes. Apparently there is a great deal of painful irritation of the extremities which may be likened to the paræsthesiæ experienced by the adult patient affected with acroparæsthesia or with erythromelalgia. The intolerable itching and prickling cause the infant or young child to bite the fingers and rub the legs together, which habit in conjunction with the sodden state of the skin often leads to the appearance of ulcers and septic blisters on the fingers and feet. At an early stage of the disease the surface epidermis of the hands and feet begins to desquamate in fine flakes. A fairly profuse nasal discharge, often of clear mucus, and a tendency to increased salivation are noticed in many cases; 
As the disease progresses the limbs become markedly hypotonic, and in chronic cases after three months or so the knee and ankle jerks are often lost, and sensory changes, of which analgesia is the most easily tested, may be observed.

In the more severe cases remarkable trophic disturbances are likely to occur. Several teeth may become loosened and even fall out. This has also been recorded as one of the earliest symptoms (Rocaz ${ }^{11}$. The gums may become detached from the alveolus. For example, in the case of an infant of eighteen months, seen by one of us, there were signs of a general improvement at about the end of the third month of illness, when to the mother's surprise one day she found that on pulling down the lower lip the whole of the gum came away from the lower jaw revealing the teeth down to their roots. The child was afebrile, but died in hyperpyrexia later in the same day. The nails have become loosened and shed in some cases. More often septic sores or ulcers form at their bases or on the palmar surfaces of the fingers and feet. Gangrene of the finger-tips has also been recorded (Parkes Weber ${ }^{7}$, Debré and Petot $^{12}$ ).

The disease runs a chronic course, often with periods of partial remission, and lasts commonly for several months to over a year. Abortive cases, however, are also recognized. As recovery takes place the capillary stasis at the extremities becomes intermittent, so that the cyanosis comes and goes, sometimes being present in one hand for a while and not in the other, and vice versa. The mortality is roughly about 5 per cent. (Muller ${ }^{13}$ ), most of the deaths being due to some intercurrent infection such as broncho-pneumonia, but occasionally the disease per se proves fatal, sometimes by cardiac failure (see Cases 4, 7).

From a clinical survey of pink disease, it is apparent that nearly all the symptoms are due to a disturbance of the vegetative nervous system (sympathetic and vagus). This important fact has been specially emphasized by Feer, who drew attention to the rise of blood pressure and of pulse rate during the disease without any corresponding rise of temperature or respiratory disturbance. The outstanding symptoms of 'vegetative' disturbance are vasomotor paralysis at the extremities, acceleration of the pulse, raised blood pressure, excessive sweating, and trophic changes. To a similar cause, in all probability, are due the photophobia without conjunctivitis, the rhinorrhœa, the sialorrhœa, and the anorexia. At a later stage of the disease evidence of lower (spinal) neuron implication is often discernible in the abolition of the tendon jerks and the presence of analgesia. In an uncomplicated case other systems than the nervous are unaffected.

Clinical pathology.-The state of the cerebrospinal fluid is normal. Blood cultures have been negative and those from the nasopharynx yield the common types of bacteria. In the blood a leucocytosis is a constant finding, granular cells predominating. The Wassermann reaction is negative. A secondary infection of the urine with bacillus coli is not uncommon. Analysis of the stomach contents revealed ' a practically normal amount of hydrochloric acid' (Byfield', 1920). 


\section{Clinical and pathological reports.}

Our investigations on the pathology of pink disease are based on the following seven fatal cases.

Case 1.-.T. N., a female child, aged 2 years and 7 months, was admitted to hospital, August 21st, 1923.

For five months previously the child had been ailing with pains in the abdomen, unaccom. panied by vomiting or diarrhoea, and with pains in the limbs. In the last two months the extremities had been noticed to be very cold and reddened, and for about six weeks there had been considerable weakness of bcth legs.

On admission the child lay flat in bed unable to sit up, and sweating was profuse. Photo. phobia, insomnia, anorexia, and increased thirst were observed. There was a striking coldness of the limbs, especially of the hands and fcet, which were bluish-red and desquamating. A

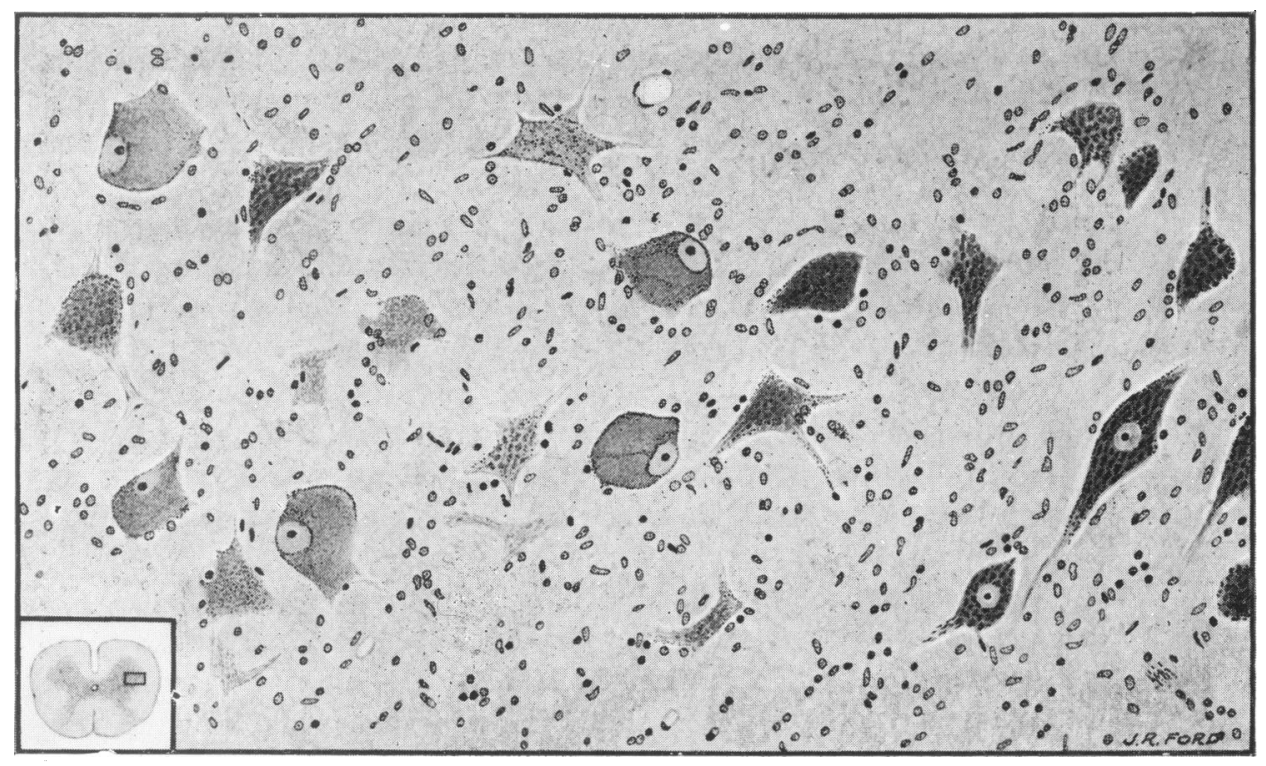

FIG. 1.-Drawing of an anterior horn of the spinal cord at the level of the fourth lumbar segment from Case 1. Stained by toluidin-blue (Nissl method). $\times 200$. Many anterior horn cells show advanced chromatolysis.

sudaminal rash covered the trunk. Small abrasions were present on several of the toes. There was a complete flaccid paralysis of both legs, but movements of the arms were fairly good. All the muscles were extremely hypotonic, and no tendon jerks could be obtained. Cutaneous sensibility was impaired over the entire body (analgesia).

The pulse rate varied between 110 and 160 ; the temperature was normal. Blood count: leucocytes $23,600,77 \cdot 5$ prr cent. being polymorphonuclear cells. Cerebrospinal fluid was normal. ('ultures :-C.S.F., negative; fæces, usual bacteria; urine, a few B. coli.

The child had one short remission with improvement, but afterwards went downhill. The muscles of the upper arms became weak, and towards the end there was a slight right facial paralysis. Swallowing was never disturbed. Death occurred from broncho-pneumonia on November 21st, 1923, after an illness lasting roughly eight months.

Avtopsy.-The body was that of an emaciated child. There were some swelling and cyanosis of the fingers, and an ulcer on the outer border of the left thumb penetrating down to the priosteum. 
The surface of the brain showed a little congestion; the spinal cord was pale and firm to touch. Numerous portions of the brain, spinal cord, peripheral nerves and vagus were taken for section. Both lower lobes of the lungs were consolidated, and the pleural cavities contained a small quantity of free fluid. Other organs, heart, liver, spleen and kidneys, appeared congested, but were otherwise normal. There was no enlargement of the lymph glands.

Microscopical EXAMination.- Sections from the thoracic and abdominal viscera appeared normal, except those from the lower lobes of the lungs which showed signs of broncho-pneumonia.

In the nervous system sections* from the cortex, pons, medulla, and cerebellum were normal. In the spinal cord at the level of the seventh cervical segment several anterior horn cells showed a slight degree of chromatolysis. In addition, there was satellitosis of some cells. In the lumbar enlargement, particularly in the fourth and fifth lumbar segments, advanced chromatolytic changes were observed in the anterior horn cells. Most of these cells were rounded and their nuclei were either grossly eccentric, lying just under the cell membrane, or had disappcared. Fig. 1 represents the condition of the anterior horn cells in the fourth lumbar segment. Satellitosis of the chromatolytic cells may be noted.

Throughout the length of the cord a slight diffuse infiltration with small round cells was present. In the lumbar region this infiltration was particularly noticeable in the anterior horns. There was no obvious increase in the number of small cells in the anterior or posterior roots.

The capillaries in the spinal cord were congested, especially those in the nerve roots of the lumbo-sacral segments.

Many nerves were stained by the Marchi-Busch method with negative results. The same nerves stained by the Weigert-Pal method exhibited varying degrees of myelin degeneration.

In the sciatic nerve some fibres were well stained. Others were poorly stained and were swollen, often showing much beading and fragmentation of the myelin. Many fibres had globular formations of degeneration products along their course. There were an undue number of non. myelinated fibres present in this nerve. This was particularly well seen in cross section. The internal saphenous nerve showed somewhat patchy staining of the myelinated fibres, but they appeared well myelinated. In the brachial plexus there were many thinly myelinated fibres. There was much fragmentation of the myelin in some fibres, and in others the myclin sheath was swollen and broken up. In the median nerve many fibres showed swelling of the myelin sheath, but most fibres were well myelinated. 'The fibres of the vagus nerve appeared healthy. The Gasserian ganglion presented no abnormalities.

The muscles of this case were comparcd with similar muscles from a child of the same age who died after a surgical operation. The fibres of the biccps brachialis in the control were of uniform size and showed distinct cross striation. The same muscle of J. N. had smaller fibres and cross striation was not so obvious. On cross section many fibres were rounded and in certain portions of the muscle there was an apparent increase in the number of sarcolemmal nuclei. In the biceps femoris the proportion of small fibres was greater than in the biceps brachialis, and cross striation was very poor. The fibres of the diaphragm appeared normal in size and cross striation was good. By the Weigert-Pal method the intramuscular nerve bundles in the muscles of the control case were stained a deep black. In the biceps brachialis and biceps femoris of .J. N., the nerve bundles stained poorly. Some of the smaller nerve bundles showed practically no myelination. In the larger nerve bundles, the myelinated ncrve fibres appeared to have swollen myelin sheaths. In the diaphragm, the nerve bundles were well myelinated.

Case 2.-P. E., a female child, aged 7 months, was admittcd to hospital, Octoler 17 th, 1924.

The infant was breast-fed. At five months she began to sleep badly, lost interest, and cricd much. A morbilliform rash appeared, covering the body, and was very irritating. Later the palms began to peel. Sweating was profuse. The bowels were regular, and respiratory catarrh absent.

On admission, the child was well nourished, but had a profuse pin-point papular rash over

*The nervous system in our seven cases was stained by various methods. All were stained by the Nissl method, hæmotoxylin and Van Gieson, Scharlach R. method for fat, and the Marchi-Busch method for recent myelin degeneration. Certain of the more chronic cases were also stained by the Weigert-Pal method, and by Anderson's Victoria blue stain for neuroglia. 
the body, and the hands and feet were cold and raw-beef colour. There were erythematous patches on the nose and cheeks. No evidence of glandular enlargement was present.

The child had some difficulty in holding up the head, and the muscles of the arms and legs were hypotonic and feeble. The knee and ankle jerks were present, and sensation was unimpaired. The pulse rate varied between 120 and 150 ; the temperature was normal until pneumonia supervened as a terminal event. Death occurred October 24th, 1924, after an illness of roughly two months.

Autopsy.- The body was that of a fairly well nourished infant. Peeling of the skin of the hands and feet was observed, and there were several small ulcers on the fingers of the right hand. The hair could be pulled out in handfuls.

The macroscopic appearance of the brain and spinal cord was normal, and the other organs showed no abnormality beyond some basal congestion of both lungs.

Microscopical examination.-Early broncho-pneumonic changes were seen in sections of the lung. Sections from the liver, spleen, kidneys, thyroid and thymus appeared normal.

The only abnormalities presented by the cerebral cortex and the brain stem were an increased vascularity of the capillaries and a slight infiltration of both the white and the grey matter with small round cells. The medulla showed a similar infiltration with small cells, but no engorgement of vessels. The cellular infiltration appeared to consist of the same type of cell as we observed in the spinal cords of this and our other cases.

In the spinal cord, one or two rounded anterior horn cells were seen at the level of the fifth lumbar segment. Otherwise the cells of the spinal cord seemed healthy. In the cervical and lumbar enlargements a diffuse infiltration of the cord with small cells was evident.

In sections of the ccrd stained by the Marchi-Busch method, fine black droplets representing degenerating myelin were seen in the dorsal roots and running into the posterior horns in the cervical enlargement. A similar appearance presented itself in the lumbar enlargement, where black droplets were seen in both the anterior and posterior roots, though more evident in the posterior roots. The dorsal region of the cord did not show any abnormal staining by this method.

The sciatic, anterior tibial, posterior tibial and vagus nerves were examined by the WeigertPal and Marchi-Busch methods, both of which gave negative results. The sensory nerves in the skin of the foot were easily discernible by the Weigert-Pal method, as they were heavily myelinated. The intramuscular nerve bundles in the biceps femoris, gastrocnemius and diaphragm were also well myelinated.

Whereas the size and shape of the muscle fibres were normal in the biceps and diaphragm, in the gastrocnemius many of the fibres were definitely smaller than in a control muscle and cross striation was not so obvious. The sarcolemmal nuclei were increased in number, especially in relation to the thin fibres.

Case 3.-M. B., a female child, aged 1 year and 11 months, was admitted to hospital, January 16th, 1925.

Six months before admission a rash appeared on the arms and legs and to a less extent on the trunk. The mouth was sore and some diarrhœa was present with a slight rise of temperature. A month later the hands and feet became swollen and reddened, and the infant was very miserable, continually rubbing the legs together. She was able to stand with support, but was weak and listless.

On admission the child was fairly well nourished, but was restless and fretful. The hands and feet were pink, swollen, and cold, and there were erythematous patches on the nose and cheeks. Fine desquamation was occurring on the cheeks, feet and hands. Photophobia was present.

The muscles of the upper and lower limbs were weak and very flabby. No tendon jerks were obtainable. There was some anæsthesia of the hands and of the legs as far up as the knees.

The inside of the mouth became ulcerated, and numerous boils kept forming on the body. The stools were loose and contained much mucus and undigested food. The temperature was raised to $100^{\circ}$. Death occurred 14 days after admission, and was due probably to toxæmia The duration of the illness was roughly six months.

Auropsy. - The body was that of a poorly nourished child, with numerous pustules on the trunk and limbs. 
Microscopical examination.-No abnormality was detected in sections of the liver, spleen. kidney, or heart muscle.

In sections of the cerebral cortex and basal ganglia, stained by hæmatoxylin and Van Gieson, a large number of small round cells were seen scattered throughout the white and gray matter. These cells were not the nuclei of fibroglia as no processes could be stained by neuroglia methods. We took them to be the same type of cell as we have described in the spinal cords of our other cases and which we also found in the spinal cord of this case.

The cellular infiltration in the spinal cord was generalized. It was present throughout the length of the cord and in both the white and the gray matter, but it was most obvious at all levels in the dorsal horns.

The anterior horn cells were normal except in the lumbar enlargement, where one or two showed eccentricity of the nucleus with chromatolysis.

The sciatic, femoral, popliteal and musculo-spiral nerves were examined by the Weigert-Pal method and by the Marchi-Busch methed. The former gave negative results, lut by the Murchi-Busch method degeneration of myelin was observed in the popliteal nerve.

No muscle was examined in this case.

Case 4.-P. M., a female child, aged 9 months, was admitted to hospital on June 8th, 1925.

Three weeks before admission the child began to draw up the legs as if in pain, rubbing the feet and hands together, and sweating profusely. The baby was very fretful and getting thin, though taking food fairly well, and was flabby, being unable to sit unsupported. The diet had been Nestlé's milk up to the seventh month, subsequently cow's milk.

On admission the child was very restless and miserable, shunned the light, and was unable to sit up. There was a papular rash over the body, and the feet and hands were typically 'pink.' The tendon jerks were present, and no sensory impairment was made out.

The pulse was usually 120 , and the temperature was normal. Death occurred suddenly two days after admission without any physical signs of pneumonia, but with a terminal temperature of $104^{\circ}$. The duration of the illness was about three months.

Autopsy.- The body was well nourished with the pink colouration of the fingers and toes still visible. No signs of pneumonia were present in the lungs. In the heart the right auricle and ventricle were dilated. The thymus was larger than normal for a nine months infant. The abdominal viscera appeared healthy. The brain and spinal cord showed no abnormality.

Mrcroscopical Examination.-Sections of the spleen, pituitary, thymus and suprarenals appeared normal.

No abnormality could be seen in sections from the cerebral cortex, or from the medulla.

In the spinal cord there was a slight excess of small cells in the white and gray matter, not confined to any special area of the cord, or more apparent at any particular level.

The anterior horn cells appeared healthy in all regions of the cord.

The sciatic, internal and external popliteal nerves were examined by the Weigert-Pal and Marchi-Busch methods with negative results. No evidence of degeneration could be observed by either method. The vagus was unfortunately not examined.

Case 5. - R. S., a male child, aged 7 months, was admitted to hospital on February 22nd, 1929.

Two weeks before admission a rash developed on the hands and arms, and in the past three days preling had occurred. The child was a full-time baby, born of healthy parents and had been quite normal up to six weeks before admission, when teething began and weight was lost. Since then there was a history of an unusual amount of crying and that the child had been observed to sit or lie with his head buried in the bedclothes. The bowels had been regular and there had been no vomiting. The child had bəen fed on the Allenbury sequence, 1, 2, 3, with occasional orange juice.

On admission the patient was found to be irritable with a red papular eruption on the hands, arms, feet, cheeks and nose. There was some preling of the nose and hands. The condition was typical of pink disease. Two teeth had erupted. The gums were swollen, but there was no stomatitis. A purulent conjunctivitis of the right eye was present, growing staphylococcus aureus on culture. The pulse rate was rapid. No definite signs could be found of involvement. of the nervous system. The reflexes were brisk and there was no definite evidence of sensory 
changes. The stools were healthy on admission, but subsequently became loose, green and fraquent, causing the buttosks to bacoms sore and excoriated. The temperature rose and the general condition rapidly becam? worse. The child died on March 4th, 1929, after an illness of about five weeks.

Autopsy.-Performed six hours after death. The body was that of a well-nourished child. There was preling of the hands and feet. Signs of a rash on the arms and feet were still present in the form of small subcutaneous red spots.

The brain and spinal cord showed no macroscopic abnormality. Portions of the median, ulnar, sciatic, posterior tibial and vagus nerves were taken for section. The heart and pericardium appeared normal. The lower lobs of the left lung was very congested, but still crepitant and there was no definite evidence of consolidation. The stomach and duodenum wers normal. The lower end of the ilium was very congested and showed a few ulcerated areas about the size of a paa which were denuded of epithelium and had raised edges. Similar areas were found scattered throughout the large bowal. The liver, spleen, kidneys, suprarenals and pancreas appzared normal.

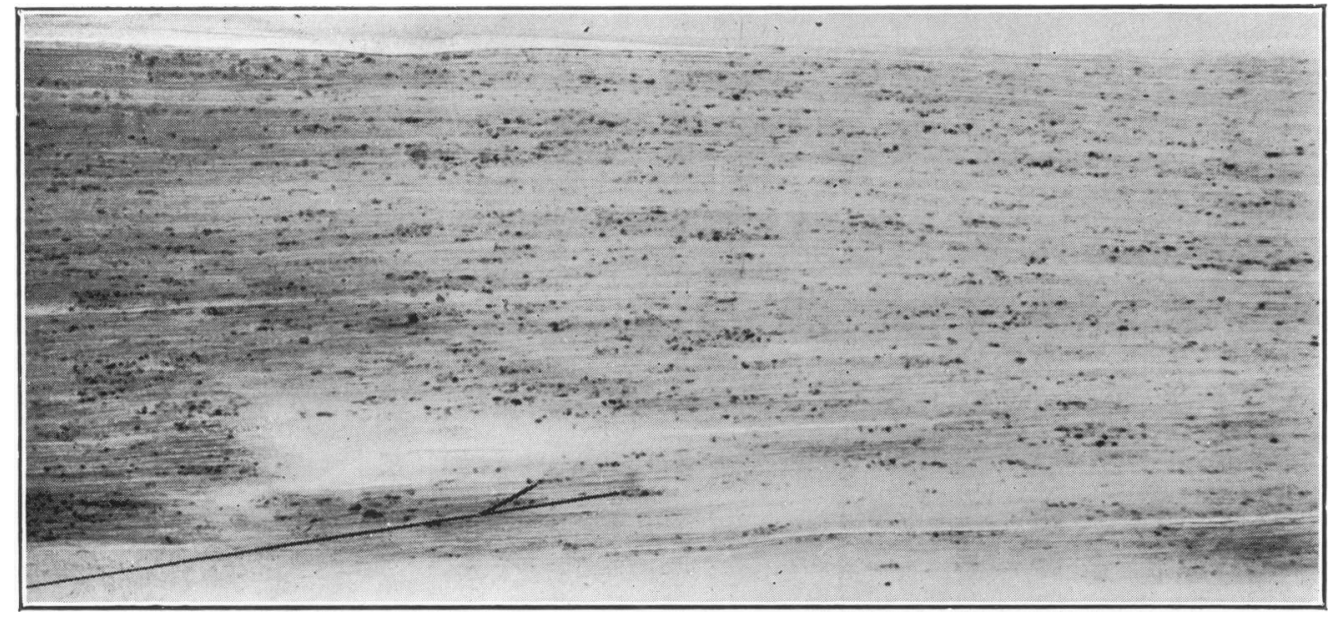

FIG. 2.- Microphotograph of a section through the median nerve of Case 5. Stained by the Marchi-Busch method. Black droplets of degenerating myelin can be seen scattered along the course of the nerve. $\times 120$.

Microscopical examination. - Sections of all the viscera appaared normal, except those from the spleen. On section this organ was more congested than normal and had an abnormally large number of red blood corpuseles in the pulp.

The carebral cortex, the brain stem and the medulla appsared normal by all staining methods.

The spinal cord, when stained with hæmatoxylin and Van Gieson's counter-stain, showed a diffuse infiltration with small pound cells in both white and the gray matter. The infiltration was most apparant in the lumbar region, but did not seem to involve the posterior roots more than the anterior roots. The infiltrating cells were not fibroglial, i.e., they showed no processes when stained with neuroglial stains. They corresponded exactly in appearance to the small round cells infiltrating the spinal cord, described by Paterson and Greenfield in their cases. They were probably lymphocytes. The Marchi-Busch method did not reveal any degeneration of roots or tracts in the spinal cord.

The anterior horn cells of the spinal cord appaared normal when stained by the Nissl method except at two levels. In the region of the cervical enlargement several rounded cells that had lost their processes were seen, and at the level of the third to fourth lumbar segment there were 
several pale cells, one cell of the antero-medial group having an eccentric nucleus and no Nissl granules.

The most striking changes denoting recent myelin degeneration in the peripheral nerves were obtained by the Marchi-Busch method. Degeneration of myelin was seen to some extent in all the peripheral nerves, but was much more evident in some nerves than in others. In the median nerve advanced degeneration was present in many fibres. No one fibre was completely degenerated throughout its length, but viewed in longitudinal section fat globules could be seen at varying distances along the course of almost every fibre (Fig. 2). In the ulnar nerve there were not so many fibres undergoing degeneration as in the median nerve, but early changes in the form of small black globules were easily recognizable. In the sciatic nerve very few fibres appaared degenerated. In the posterior tibial nerve many fibres in each nerve bundle were seen to be in a fairly advanced stage of degeneration. In the vagus nerve the great majority of the fibres appeared normal, but one or two showed early degeneration.

The nerves when stained with hæmatoxylin and Van Gieson's counterstain presented no abnormalities. There was no cellular infiltration and no thickening of the neural sheaths.

The fibres of a portion of the biceps femoris muscle were entirely normal in size and structure.

Case 6.-B.G., a female child, aged 7 months, was admitted to hospital on August 14th, 1929.

The history from the mother was that the child had lost weight from the age of 5 months, even while still breast-fed. She had been fed at the breast until the age of 5.12 months and had then been given cow's milk with egg yolk and orange juice. There had been severe loss of apprtite and the child had bren sleeping badly. The bowels had been loose when the symptoms first appaared, but had since been constipated.

On admission the pxtient was seen to be a restless crying child. The back and front of the chest and the abdomen were covered with a fine erythematous rash which appeared to be irritating. On the chest also were one or two ulcerating septic spots. The hands were red and covered with the same rash; the skin of the hands seemed to be slightly puffy. The fingers were peeling. Just above the left ear was a hard inflamed swelling, about two inches in diameter, which was full of pus. This was aspirated and a perchloride dressing applied.

There was marked loss of tone in all the muscles, more particularly in those of the thigh and uppær arm. The knee-jerks, however, were present. There was apparent impairment of sensation over the feet. The apex beat was rather diffuse, but strong, and was felt in the fifth and sixth spaces, inside the nipple line.

The child was very restless and spent most of her time with her face buried in the pillow. Two days later she was much quieter, but listless. Another septic boil appeared on the back of the head, and two others on the back. These were opened next day, but the child died the following day after an illness of roughly two months. The pulse rate at death was 160, temperature $103 \cdot 6^{\circ}$.

Autops r.--Performed 26 hours after death. The body was that of a poorly nourished ehild. The legs were sedematous. The thumb and inner two fingers of the right hand were desquamating and a vesicle was present on the big too of the left foot. A rash was present on the buttocks, and sites of abscesses could bo seen behind the left ear and at the back of the head.

The thymus extended downwards as far as the upper border of the heart. The right ventricle and auricle contained post-mortem blood clot. The heart was otherwise healthy. In the abdomen a large number of enlarged soft mesenteric glands were present. These glands were of normal consistency. The liver was fatty but not flabby. The spleen was firm and the Malpighian corpuscles stood out plainly. Both kidneys were pale and the left one showed fœtal lobulation. The stomach contained coffee-ground material and mucus. The nesophageal opening was injected and showed petechial hæmorrhages. Otherwise there was no evidence as to the origin of the blood found in the stcmach.

The brain and spinal cord presented no macroscopic abnormality. Several peripheral nerves were removed for histological investigation.

Microscopical Examisation.-. Sections of both lungs showed the typical early bronchopneumonic changes of catarrh of the bronchi with polymorphonuclear exudate. Liver :--slight 
fatty degeneration was present in the liver cells. Kidneys :-cloudy swelling was seen in the convoluted tubules. Sections of the spleen and intestine appeared normal.

In the skin, hyperkeratosis was the outstanding lesion. We did not observe any cellular infiltration. There were a very large number of sweat glands present in the portion of skin examined, probably an excess of the number normally present.

All parts of the nervous system above the level of the medulla appeared normal.

In the spinal cord the anterior horn cells were healthy at most levels, but at about the second or third lumbar segment a few cells of the anterior group had eccentric nuclei and were rounded and pale, with their Nissl granules clustered irregularly around the nucleus. A generalized infiltration with small round cells was found throughout the cord, but this infiltration was greatest in the lumbar region, where, in one or two sections, a special accumulation of cells was present at the entry zone of the posterior roots. The character of the infiltrating cells was the same as those abserved in our other cases.

The sciatic, external popliteal, dorsalis pedis and musculo-spiral nerves all showed early degeneration of their fibres by the Marchi-Busch method. The degeneration was not wide spread

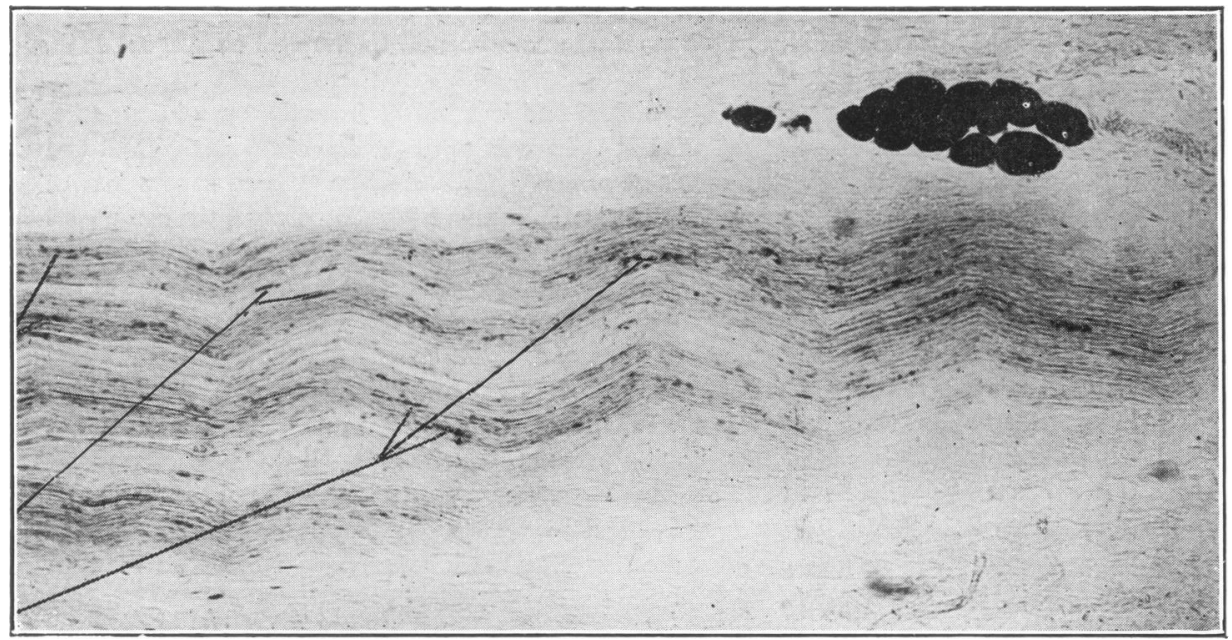

Fig. 3.-Microphotograph of a longitudinal section through the vagus nerve of Case 7 . A number of black droplets representing degeneration of myelin may be seen. $\times 12$.

in the nerves, but was confined to parts of the fibres, being always more advanced and more apparent at the nodes of Ranvier. No degeneration was seen in the phrenic nerve.

When stained by hæmatoxylin and Van Gieson, none of the nerves exhibited any cellular infiltration, but there was a slight proliferation of the nuclei of the sheath of Schwann.

No muscle was examined in this case.

Case 7.-D. B., a male child, aged 1 year and 3 months, was admitted to hospital on May 23rd, 1930.

The history obtained was that the child had been perfectly healthy up to one month before admission when he seemed to lose strength, ceased to walk and to talk, refused his food, became very irritable, and developed a sore mouth. He had been breast-fed for three months, was then given Savory and Moore's food until nine months of age and since had been on a mixed diet.

On admission the patient was found to be a miserable child with flabby muscles. There was marked stomatitis and the teeth, though healthy, were all loose. The skin of the hands and feet was pink and the condition typical of pink disease. The knee-jerks were present. The pulse rate, already high, rose to 164 par minute the day after admission, when the child collapsed and died suddenly. The length of the illness was roughly one month. 
Autopsy.-Performed 48 hours after death. Traces of a pink eruption were still present on the hands and feet. No macroscopic abnormality was seen in the brain or spinal cord. Portions of many nerves were taken for section. The lower lobes of both lungs were congested. 'The heart muscle was contracted : death had occurred in systole. There was no dilatation of the right side of the heart. The muscle was not abnormally pale or easily friable. The stomach was large and atonic. There were slight inflammatory changes in the stomach wall. The intestines appeared normal. The liver showed a moderate degree of mottling due to fatty change. The spleen, kidneys and suprarenals appeared healthy.

Microscopical examination.-Sections of the heart muscle, thymus, lung and spleen appзared normal. In sections of the liver cloudy swelling and early degeneration of the tubular epithelium were seen. There was a slight degree of fatty change in the liver cells.

The cerebral cortex, mid-brain, pons and medulla appeared normal.

The whole length of the spinal cord was not available for section, but portions were taken from the lower cervical, mid-thoracic and mid-lumbar regions. The anterior horn cells in sections made at these levels showed no evidence of chromatolysis or other abnormality. There was a slight excess of small cells in both the white and gray matter of the portions of the spinal cord which we examined, but this cellular infiltration was less than in our other cases.

The sciatic, posterior tibial and cervical sympathetic nerves did not present any evidence of degeneration when stained by Scharlach $R$. and by the Marchi method. In the vagus nerve, a number of fibres showed definite degenerative changes by both these methods. With the Scharlach R. stain we observed swelling of the nerve fibres and fragmentation of the myelin. The picture exhibited by the Marchi-Busch method is represented in Fig. 3. It will be seen that no one fibre has degenerated throughout its course, but that many fibres have begun to degenerate in part of their course.

No muscle was examined in this case.

\section{Pathology.}

The immediate cause of death in pink disease is usually an intercurrent infection, such as broncho-pneumonia, or a concomitant disease, such as tuberculosis. In Wood's series of 91 cases there were five deaths, of which four were due to broncho-pneumonia. The pathological picture, therefore, of pink disease is apt to be complicated by the morbid histology of a secondary disease process. It is for this reason that the pathological examination of the nervous system is of great importance, as the changes which have been reported in the nervous system in certain cases of pink disease cannot be ascribed to the terminal complications of the disease. They depend upon the disease itself.

The pathological findings which Wood reported in three of his cases were essentially those of broncho-pneumonia. The nervous system was not examined pathologically. No autopsy was performed on the fifth patient, who died suddenly of heart failure. Byfield was one of the first to examine the nervous system. His case was a relatively acute one complicated by tuberculosis, which produced characteristic lesions in the lungs. In the spinal cord there was degeneration of an occasional anterior horn cell, cedema of the sensory roots, and also of the myelin sheath of the sciatic nerve, which is a very early stage in the degeneration of the nerve.

Greenfield and Paterson ${ }^{13}$ in this country were the first to make a thorough examination of the nervous system in pink disease. Their cases are especially important from the point of view of comparison between complicated and uncomplicated cases. One case was uncomplicated 
and recovery was slowly taking place when the child died suddenly from an intussusception, whilst the other child went slowly downhill and died from generalized tuberculosis. The symptoms of pink disease had been present 3 or 4 months in the first and about 8 months in the second case. In both the same lesions were found in the nervous system, though they were more intense in the long-standing case in which death occurred from tuberculosis. In both cases degeneration of myelin was found in the peripheral nerves of the limbs, more extensive distally than proximally. The vagus and cervical sympathetic nerves were normal. The calf muscles in one case were atrophied, and the small nerve bundles in the substance of the muscle showed degeneration. Above the medulla the nervous system appeared normal. A diffuse infiltration with small cells, which the authors considered to be glial rather than hæmatogenous, was present in the spinal cord in both cases, particularly in the lumbo-sacral region, and there was also an increase of the cellular elements in the nerve roots in the same area. In the more chronic case, especially in the cervical and lumbo-sacral region, the ventral horn cells showed an 'axonal reaction' with central chromatolysis, eccentricity of the nucleus and vacuolation of the cytoplasm.

Feer ${ }^{9}$ reported one case with an autopsy. His case was complicated by a terminal bronchopneumonia, and post mortem the lesions of that condition only were found. The spinal cord and sympathetic system were examined, but not the peripheral nerves.

Butler ${ }^{15}$ performed a biopsy and removed a piece of skin from the hand of one case. His description of the skin lesion is of interest. He found hyperkeratosis with ballooning of the cells of the granular layer, and lymphocytic infiltration of the corium with much œdema. He pointed out that nothing in this report was pathognomonic of pink disease, although the outstanding feature, the œdema of the corium, is an unusual finding.

Warthin ${ }^{16}$ made a complete examination of two cases in which the cause of death was broncho-pneumonia. He also gave a detailed description of the skin lesion. In both cases the skin showed hyperkeratosis with a scaly desquamation of the horny layer. The papillæ were enlarged, and the perivascular reticulo-endothelium was hyperplastic, but there was no cellular infiltration and only slight œdema. The sweat glands were dilated and hypertrophic, and the sebaceous glands were very small. In the thymus the lymphoid tissue was atrophied and the germ centres in the spleen showed lymphoid exhaustion. The pituitary and pineal glands were normal. The only changes which Warthin found in the nervous system were proliferation of the reticulo-endothelial cells in the cerebral meninges, and congestion of the meninges and brain substance.

Francioni and Vigi ${ }^{17}$ recorded an interesting case, that of a child of four and a half years who developed pink disease within a few weeks of an attack of epidemic encephalitis. The autopsy and subsequent microscopical investigation revealed the typical appearances of epidemic encephalitis with, in addition, an infiltration of the cervical sympathetic ganglia with small lymphocytes. No peripheral nerves were examined. The views of the authors on the interpretation of their findings in this case will be referred to later in discussing the theories of pathogenesis.

Kernohan and Kennedy ${ }^{18}$ examined in great detail a case in which the cause of death was broncho-pneumonia. The visceral changes were solely those associated with the terminal condition. In the nervous system they describe extensive degenerative changes. These are especially striking as the recorded duration of symptoms was only three weeks. The Marchi method demonstrated myelin degeneration in the femoral and sciatic nerves, and a little also in the brachial plexus. In the sacral, and to a less extent in the lumbar region of the cord, chromatolysis of the ventro-lateral group of anterior horn cells was evident. Varying degrees of chromatolysis were recorded in the cells of the dorsal root ganglia, particularly in those from the lumbar region. The changes varied from slight eccentricity of the nucleus and loss of Nissl granules to complete disintegration of cells. In the brain chromatolytic changes were found in the cells of the eminentia teres, the mesencephalic root of the fifth nerve, the locus cæruleus, the lenticular nuclei and in the anterior thalamic nuclei. The cells of the Gasserian ganglia also showed extensive chromatolytic changes. Some of these findings are undoubtedly open to criticism for reasons which will shortly be mentioned.

Findlay and Stern ${ }^{19}$ who produced in rats a condition clinically and pathologically resembling pink disease also published a report of the pathological investigation of a typical mild case of the 
disease. The child died from tuberculous broncho-pneumonia. On histological examination the viscera showed only the lesions attributable to that complication. In the nervous system, however, there was found a diffuse infiltration with small round cells, most evident in the cervical region and in the lumbo-sacral enlargement. The infiltration extended along the posterior nerve roots, but the anterior nerve roots were unaffected. A similar infiltration was seen in the medulla. Early chromatolytic changes were present in the eighth cervical segment and in the dorso-lumbar enlargement, and early myelin degeneration was found in the sciatic nerves. The cervical and abdominal sympathetic ganglia and the carotid ganglia were normal. No abnormalities were detected in the muscles, and the intramuscular nerve fibres appeared healthy.

The histological examination of our seven cases of pink disease does not present any new features. It merely confirms those which have already been recorded, and serves to illustrate that lesions in the nervous system occur in a high proportion of cases. Degenerative changes were found in the peripheral nerves in four out of seven cases. In one case of very long standing (Case 1), myelin degeneration had reached such an advanced stage that the Weigert-Pal method was applicable. In another case (Case 7), an interesting feature was present. The child died suddenly of heart failure after a short illness, and the only nerve in which degeneration was found was the vagus.

The spinal cords in all seven cases showed the diffuse infiltration with small cells first described by Paterson and Greenfield. It was very distinct in five cases and slight in the other two. We were unable to determine the nature of the infiltrating cells. They resemble glial cells rather than lymphocytes in their staining reactions, but we could not demonstrate processes on them by either neuroglial or microglial staining methods.

Chromatolysis of the central type was found in the anterior horn cells of the spinal cord in five cases and it was more apparent in the lumbo-sacral region than elsewhere. It was extensive in the most chronic case and was clearly due to an 'axonal reaction.'

Slight changes in the muscles were observed in two cases. These changes were those of a simple atrophy consequent upon a neural lesion.

In only two cases could we detect any abnormality in the nervous system above the level of the medulla. In Case 2 a slight cellular infiltration was present in sections of the cerebral cortex, brain stem and medulla; in Case 3 a similar infiltration was present in the cerebral cortex and the basal ganglia. In the other cases the infiltration was confined to the spinal cord and nerve roots. The cortex, mid-brain, pons and medulla were carefully sectioned for evidence of chromatolysis of nerve cells, but with negative results.

Discussion.-From a consideration of the foregoing pathological examinations the essential lesions in pink disease would appear to lie in the skin and in the nervous system. The lesion in the skin is, according to Butler, not pathognomonic. He described exactly similar changes in the skin in a case of acromegaly. In the nervous system, the evidence for a characteristic and pathognomonic lesion is somewhat meagre, but in the majority of cases in which the peripheral nerves have been examined, evidence of some degenerative change has been forthcoming.

Chromatolytic changes in the anterior horn cells of the spinal cord may be due to a large number of causes, but the fact that these changes in pink 
disease occur in the spinal segments corresponding to the nerve supply to the limbs suggests that such chromatolytic changes are due solely to an 'axonal reaction.' The significance of the cellular infiltration of the spinal cord and nerve roots which occurs in a proportion of cases cannot as yet be explained.

The association of a cellular (lymphocytic) infiltration of the cervical sympathetic ganglia with the characteristic changes of epidemic enoephalitis in the mid-brain, which Francioni and Vigi described in their case, is of interest in view of the theories of pathogenesis to be mentioned later.

The extensive chromatolytic changes in certain nerve cells which Kernohan and Kennedy described call for some comment. Those changes which were observed in the anterior horn cells of the spinal cord and in the spinal ganglia may be accepted without reserve, and form a valuable addition to our knowledge of the lesions in pink disease. The chromatolysis, however, described in other parts of the nervous system, particularly in the cells of the eminentia teres, the mesencephalic root of the fifth nerve and in the thalamic nuclei cannot be so accepted. Nerve cells in these areas of the brain in a normal subject were subjected by us to a careful scrutiny and we were unable to convince ourselves that rounded cell bodies, eccentric nuclei and peripherally placed or clumped Nissl granules were not normal to cells in these situations. We also referred to the volume on the nervous system in the recent 'Handbuch der Microscopischen Anatomie der Menschen,' in which the descriptions and pictures of cells in the areas of the brain under consideration confirmed our observations that the cells in these areas do not conform to the common type of pyramidal nerve cell with a central nucleus and evenly distributed Nissl granules. We suggest, therefore, that until further evidence of the degenerative changes which Kernohan and Kennedy have described in scattered areas of the brain is brought forward, the question whether these changes should be accepted as part of the pathological picture of pink disease should be left open. We were unable to confirm their results from examination of our own cases.

It is here convenient to summarize the chief clinical and pathological features of our seven fatal cases :-

Case 1.-J. N., female, aged 2 years 7 months. Duration of illness 8 months. No prodromal symptoms. Knee jerks absent. Cellular infiltration of spinal cord. Degeneration of peripheral nerves.

Case 2.-P. E., female, aged 7 months. Duration of illness 2 months. No prodromal symptoms. Knee jerks present. Cellular infiltration of cerebral cortex, brain stem, medulla and cord. No degeneration of peripheral nerves.

Case 3.-M. B., female, aged 1 year, 11 months. Duration of illness 6 months. Knee jerks absent. Prodromal diarrhœa. Cellular infiltration of cerebral cortex, basal ganglia and spinal cord. Degeneration of peripheral nerves.

Case 4.-P. M., female, aged 9 months. Duration of illness 3 months. Knee jerks present. No prodromal symptoms. Died suddenly with rapid pulse. Cellular infiltration of the spinal cord. No degeneration of peripheral nerves.

Case 5.-R. S., male, aged 7 months. Duration of illness five weeks. No prodromal symptoms. Knee jerks present. Cellular infiltration of the spinal cord. Degeneration of peripheral nerves.

Case 6,-B. G., female, aged 7 months. Duration of illness 2 months. Prodromal 
symptoms, looseness of motions. Knee jerks present. Cellular infiltration of spinal cord. Degeneration of peripheral nerves.

Case 7.-D. B., male, aged 1 year 3 months. Duration of illness 1 month. No prodromal symptoms. Knee jerks present, Cellular infiltration of spinal cord. No degeneration of peripheral nerves, but degeneration in vagus nerve.

Summary.-A point which appears to need comment is that in all these cases the clinical symptoms were much in excess of the pathological findings, although cellular infiltration was present in all seven and degeneration of peripheral nerves was found in four. Two children died suddenly with a rapid pulse which suggested vagal inhibition. In one of these (Case 7), the vagus nerve were examined and found to be degenerating. The only prodromal symptom observed was diarrhœa, which occurred in two cases. Case 5 is of particular interest in view of the short history (five weeks) and the pathological finding of degeneration in the peripheral nerves.

\section{Pathogenesis.}

Soon after the recognition of the clinical entity which we now call pink disease, two theories of its pathogenesis were advocated. One that the condition was consequent upon an unknown vitamin deficiency, and the other that it was the sequel to an infection whose nature and portal of entry were as yet undetermined. Wood in his earlier paper (1921) did not, even on the basis of his ninety-one cases, venture to give an opinion on one or the other of these theories, but in a later communication ${ }^{20}$ (1927) he had come to the conclusion that the disease was not an avitaminosis. He agreed, however, with Warthin that the condition was closely related to pellagra, or that it might be an infantile form of that disease. Goldberg's work on pellagra and its causation from deficiency of vitamin B2 had not at that time been published. For this reason it may be assumed that Wood subscribes to the avitaminosis theory rather than to the theory of an infective origin. Others who maintain that pink disease is due to a vitamin deficiency are McClendon ${ }^{21}$ and Zahorsky ${ }^{22}$, both of whom claimed good results from treatment with yeast and irradiated ergosterol.

A view slightly divergent from the avitaminosis theory is held by Findlay and Stern ${ }^{19}$ who, as the result of experimental work on rats suggest that the absence of a dietetic factor other than the known vitamins is responsible for the causation of pink disease. They produced a condition in young rats clinically resembling pink disease by feeding them with dried egg white as their sole source of protein, the rest of the diet being adequate and rich in vitamins. The same condition was produced in breast-fed rats by feeding the mothers on a similar diet. Cultures from the affected animals were negative. Attempts to reproduce the disease by means of intraperitoneal or intracerebral injection of blood or emulsions of the spinal cord from the affected animals were unsuccessful. Pathologically, degenerative changes were found in the peripheral nerves and the same curious cellular infiltration of the spinal cord that has been described in pink disease was observed in the cords of the rats, 
Although the formation of a toxic substance during the drying of the egg white must be considered as a possible positive factor, the experimental evidence certainly suggested that an essential factor in the diet was lacking, especially as the condition was rapidly cured by the addition of raw liver to the diet. The authors tentatively suggested that if the condition which they produced experimentally was identical with pink disease, then a dietetic factor in addition to the known vitamins is necessary for normal nutrition in the young child.

The consensus of opinion, however, in this country, in America and in France, is that pink disease is due to an infection. Paterson and Greenfield ${ }^{14}$ were the first to bring forward that theory in England, although Byfield had previously drawn attention to the similarity of the polyneuritic changes in pink disease to those in post-influenzal polyneuritis and radiculitis.

Rodda ${ }^{23}$, in support of the infective theory, maintained that he had never seen a case of pink disease in which there had not been a previous focal infection of the upper respiratory tract. Vipond ${ }^{24}$, Péhu and Ardisson ${ }^{25}$, Littlejohn ${ }^{4}$, and Kernohan and Kennedy ${ }^{18}$, also regarded the naso-pharynx as the portal of entry of the infective agent. Kernohan and Kennedy considered that the changes in the nervous system, which they described, were toxic in origin, secondary to dissemination of the products of the infective agent throughout the body.

The nature of the infective agent has been the subject of much speculation, especially in France. Janet ${ }^{26}$ and Turquety ${ }^{27}$ maintained that the infection was probably due to a neurotrophic virus, which Janet thought was allied to the virus of epidemic encephalitis, although Turquety did not agree with this view. Both these authors considered that the virus gained access to the nervous system through the naso-pharynx, and that it probably exerted its effects on the vegetative centres in the mid-brain.

Francioni and $\mathrm{Vigi}^{17}$, to whose paper reference has been made, were also of the opinion that pink disease was due to a neurotrophic virus which entered the nervous system through the naso-pharynx. They believed that the virus was either identical with, or closely allied to, that which produces epidemic encephalitis, and that definite and unmistakable lesions were produced in the vegetative centres of the infundibular region. Their case in which pink disease developed a few weeks after a typical attack of epidemic encephalitis certainly had lesions in these centres as well as cellular infiltration of the cervical sympathetic ganglia. It is, however, premature to base a theory on one such case, as the lesions of epidemic encephalitis itself may be scattered throughout the nervous system and despite the ubiquity of epidemic encephalitis, theirs is the first and only case we have been able to trace in the literature in which the symptoms of pink disease have been associated with an attack of epidemic encephalitis. Further pathological evidence, therefore, is needed before the theory of a neurotrophic virus allied to that of epidemic encephalitis can be seriously considered as the cause of pink disease.

A theory which has received scant attention as yet, but which accords well with the mass of clinical evidence, is that brought forward by Feer and - Selter in Germany. Both these authors regarded the condition as a 
'trophodermatoneurosis,' dependent upon a dysfunction of the vegetative nervous system. They instanced the cold clammy extremities, the sweating, the rapid pulse, the high blood pressure and the irritability of the affected children as evidence of a disturbance of the sympathetic system and compare the rash with those of other known dermato-neuroses. Feer had the sympathetic system ' examined minutely' in a fatal case of pink disease, but no organic changes were found. He therefore came to the conclusion that the condition was due to a disturbance of function and not to one of structure. This theory is worthy of more attention in view of the fact that the structural changes in the nervous system are often slight in comparison to the severity. of the clinical symptoms.

Which of the various theories of pathogenesis best explains the production of the lesions in the nervous system is a difficult question to answer. A deficiency of any of the known vitamins can be excluded as the cause, as in most cases, in which the diet has been investigated, they have been present in adequate quantity. Cases with prodromal symptoms of pharyngeal or intestinal catarrh favour an intective origin, but in numerous instances, as in several of our own cases, no history of preceding infection could be elicited. In none of our cases was there any evidence of an inflammatery cervical adenitis.

\section{Treatment by liver.}

With a special diet, already referred to, Findlay and Stern ${ }^{19}$ (1929) were able to produce in young rats a syndrome resembling pink disease both in its clinical and anatomical aspects. Adult rats had a much greater resistance against the development of the disease, but the milk of female rats so fed caused the disease to appear in their sucklings. By adding raw liver to the diet it was found that the disease in rats could be rapidly cured. Acting on this suggestion of a similarity between the human and animal syndromes, raw liver was given in human cases of pink disease with satisfactory results.

Case 8.-I. W., a female child, aged 1 year and 5 months, breast-fed for four months. The infant had a five months history of irritability, and wasting, with an itchy eruption, sweating and desquamation of the skin. She was miserable, whining, shunning the light, and burrowing her head in the pillow. The hands and feet were typically raw-beef in colour, the eyelids were inflamed, nasal discharge was present, and there were numerous septic sores on the hands and feet. The pulse rate was 125 to 140 , the systolic blood pressure $110 \mathrm{~mm}$., and the temperature normal. Analgesia was present as tested by pin-prick, the knee jerks were absent, and the muscles extremely hypotonic.

Raw liver, two ounces pounded and mixed with milk, or with a drachm of port-wine to the ounce, was added to the diet as a daily ration. Within a week a remarkable change occurred. The child was able to sit up in bed, contented, taking notice, and photophobia was much less. The rash disappeared, and the inflammation of the eyelids and the septic sores on the hands and feet quickly healed. The swelling and the raw-beef colour of the hands and feet were much improved. It appeared that the activity of the disease had become arrested, though several more weeks elapsed before a slight residual cyanosis of the hands and feet disappeared. The child was kept under observation for six weeks on liver diet. When discharged the knee jerks were still absent, but the child was otherwise in good health. 
Case 9.--J. S., a female child, aged 7 months, and breast-fed. There was an eight weeks history of anorexia, loss of weight, irritability, photophobia, profuse sweating, and an erythe. matous rash on the hands and feet. The knee jerks were absent, and there was no sensory impairment.

Raw liver, one ounce a day, was given. On the fifth day of treatment there was some improvement, on the ninth day the child was definitely better, and on the twentieth day only some hypotonia of the muscles and slight cyanosis of the hands remained.

Case 10.-M. R., a female child, aged 1 year and 4 months, breast-fed for nine months. For four months the infant had been miserable, wasting, without appetite, avoiding the light, and sweating profusely. The muscles were hypotonic and the hands and feet a typical raw-beef colour and very cold. There had been no respiratory catarrh. The pulse rate was between 110 and 140, and the temperature normal. The knee jerks were obtained with difficulty.

The case is interesting because an attempt was made to give the liver treatment at home. Raw liver, $2 \frac{1}{2}$ ounces, was ordered to be given daily, and in 3 months no improvement had occurred. The treatment had apparently completely failed, but as considerable doubt existed whether instructions had been properly and regularly carried out, the child was admitted to hospital for a further trial. Raw liver, 2 ounces daily, was then given for 14 days, in which period the child became cheerful and all the symptoms disappeared except slight photophobia and a little duskiness of the hands and feet. As improvement occurred, the cyanosis of the extremities became phasic, coming and going in the hands and feet several times in the same day.

The effect of liver treatment in this case is doubtful, as the child might have been expected to recover spontaneously in eight months, yet the rapidity with which the symptoms improved while under observation on liver treatment was very noticeable.

Case 11.-H. M., a male child, aged 7 months, cut two teeth at five months. At five months the infant became miserable, crying continually, without appetite, and losing weight. In bed he assumed the knee-elbow position with the head burrowed in the pillow. Nasal catarrh, photophobia, sweating, and coldness and redness of the hands and feet were present. Pulse rate 115 to 130 , temperature normal, knee jerks present.

30.1.31. Raw liver, 2 ounces daily, mixed with milk and flavoured with sugar, was commenced. Weight : $12 \mathrm{lb} .11 \mathrm{oz}$.

14.2.31. Erythema of feet and hands improved. Photophobia much less. Disposition more contented. Weight: $13 \mathrm{lb} .4 \mathrm{oz}$.

4.3.31. Pink condition much improved.

13.3.31. Complete recovery. Weight: $14 \mathrm{lb}$.

Recovery in this case took place in 6 weeks, which was at a slower rate than in Cases $\mathbf{1}$ and 2.

Case 12.-A. S., a female child, aged 5 years and 10 months (Dr. Cockayne's case). This child was not admitted to hospital and only seen at fortnightly intervals.

There was a three weeks history of pains in the limbs, hands, groins and jaws. The child was miserable and losing weight.

4.9.30. Temperature $99 \cdot 4^{\circ}$. No physical signs of disease were found.

25.9.30. The child was very miserable, restless, and complained of pains in the hands. A miliary rash was present on the lower part of the trunk, and the feet and hands were cold and raw-beef colour. There was general muscular atony, and the knee-jerks were absent. The appearances were typical of pink disease.

From this date, liver soup, half a pint a day, was commenced. When next seen, fourteen days later (9.10.30), the child was much better, less irritable, and sleeping well. The colour of the extremities had improved and they were no longer cold. The left knee jerk was present, but feeble. The right knee jerk absent.

23.10.30. There was no evidence of pink disease present, except that the right knee jerk was still absent.

A subsequent note five months after the child first came under observation reads that both knee jerks were obtainable, but diminished.

While these five cases were under observation, no other special form of treatment was given. Cod-liver oil and orange juice were included in the diet, 
but had already been given at home in the two infants aged seven months. In previous cases we have never seen any material benefit occur from the inclusion in the diet of substances containing any of the known vitamins $A, B$, $\mathrm{C}$, and D.

The fairly rapid improvement witnessed in Cases 8, 9, 11 and 12 speaks well, in our opinion, for liver therapy. It appeared to arrest the activity of the disease within a short time, a week to fourteen days, although a considerably longer period is necessary for such signs as the cyanosis of the extremities and the photophobia completely to disappear. A return of the knee jerks, as might well be expected, was delayed the longest. One of the earliest effects of liver therapy was a noticeable improvement in the general condition of the patient: the irritability and pain apparently quickly subsided. None of the cases relapsed.

Five cases is too small a number on which to base emphatic views, but from the results we have obtained with liver therapy, we submit that a dietetic factor contained in liver has a definite value in curing the disease. Of the nature of this factor, and whether a deficiency of it can cause the disease in the absence of a positive factor, possibly infective, we are at present unable to judge.

We desire to express our thanks to members of the honorary staff of the Hospital for Sick Children, Great Ormond Street, for placing the pathological material at our disposal and for their permission to publish the cases; to Dr. Paterson and Dr. Sheldon for two cases from Westminster and King's College Hospitals respectively. Finally, we wish to thank Dr. J. Godwin Greenfield for granting us facilities for studying our material in the laboratory of the National Hospital, Queen Square, for his interest in the work, and for kindly taking the microphotographs.

\section{REFERENCES.}

1. Selter, P., Arch. f. Kinderh., Stuttgart, 1926-1927, LXXX, 244.

*2. Swift, H., Australasian Med. Cong., Children's Sect., 1914.

3. Wood, A. J., Med. J. Australia, Sydney, 1921, I, 145.

4. Littlejohn, E. S., Idem., 1923, I, 689.

5. Byfield, A. H., J. Am. Med. Ass., Chicago, 1917, LXVIII, 1851 ; Am. J. Dis. Child., Chicago, 1920, XX, 347.

*6. Bilderback, J. B., Northwest Med., Seattle, 1919, XIX, 263.

7. Weber, F. Parkes, Brit. J. Derm., Lond., 1921, XXXIII, 228.

8. Thursfield, J. H., \& Paterson, D., Brit. J. Child. Dis., Lond., 1922, XIX, 27.

9. Feer, E., Ergeb. d. inner Med. u. Kinderh., 1923, XXIV, 100 ; Jahrb. f. Kinderh., Berlin, 1925, CVIII, 267.

10. Haushalter, P., Rev. Neurol., Paris, 1925, I, 401.

*11. Rocaz, M., quoted by R. Debré et Servel de Cosmi. Le Monde Méd., 1930, XL, 33.

12. Debré, R., \& Petot, Cl., Presse méd.. Paris, 1927, XXXV, 753. 
13. Müller, H., Arch.f. Kinderh., Berlin, 1927, LXXXI, 81.

14. Greenfield, J. G., \& Paterson, D., Quart. J. Med., Oxford, 1923, XVII, 6.

15. Butler, J., Arch. Dermat. and Syph., Chicago, 1925, XI, 166.

16. Warthin, A. S., Arch. Path. and Lab. Med., Chicago, 1926, I, 64.

17. Francioni, C., \& Vigi, F., Riv. Sper. di Fren., 1928, LII, 307.

18. Kernohan, J. W., \& Kennedy, R. L. J., Amer. J. Dis. Child., Chicago, 1928, XXXVI, 341.

19. Findlay, G. M., \& Stern, R. O., Arch. Dis. Child., Lond., 1929, IV, 1.

20. Wood, A. J., Med. J. Australia, Sydney, 1927, II, 506.

21. McClendon, S. J., J. Am. Med. Ass., Chicago, 1929, XCIII, 455.

22. Zahorsky, J. Am. J. Dis. Child., Chicago, 1929, XXXVII, 449.

23. Rodda, F. C., Idem., 1925, XXX, 224.

24. Vipond, A. E., Arch. Ped., N.Y., 1922, XXXIX, 699.

25. Péhu, M., \& Ardisson, P., Paris méd., Paris, 1926, II, 371.

26. Janet, H., J. de Méd. et de Chir. Prat., 1928, XCIX, 885.

27. Turquety, R. J., Méd. franc., 1929, XVIII, 272.

( $*$ Not consulted in the original.) 